\section{Neue Glukokortikoid-Generation: Zielort Nase}

W ährend HNO-Ärzte eine akute eitrige Sinusitis üblicherweise mit Antibiotika und abschwellenden Nasentropfen behandeln, besteht bei der chronischen Sinusitis keine durch Studien abgesicherte internationale Behandlungsrichtlinie, bemängelt Claus Bachert (HNO-Klinik der Universität Gent). So sei, wie er auf einem Symposium ausführte, der Wert einer Antibiose umstritten, ihre Dauer nicht einheitlich und die Frage der adjuvanten Therapie mit oralen oder topischen Steroiden nicht abschließend beantwortet.

\section{Steroide sowohl bei Sinusitis ...}

Bei der Behandlung einer Sinusitis kommt es laut Bachert darauf an, die $\mathrm{Na}$ senschleimhaut, insbesondere den ostiomeatalen Komplex, abzuschwellen und den Entzündungsprozess zu stoppen. Plazebo-kontrollierte, randomisierte Doppelblindstudien mit mehr als 2.500 Patienten hätten gezeigt, dass sowohl bei akuter als auch bei akut rekurrierender Sinusitis neben antibiotischer Behandlung die Gabe eines topischen Steroids die Symptome wesentlich verringern kann. Unter Langzeittherapie mit täglich $400 \mu \mathrm{g}$ Mometason habe auch das Auftreten neuer Sinusitis-Rezidive um mehr als zwei Monate hinausgezögert werden können. Aufgrund dieser Studien muss, so Bachert, der bisherige Therapieansatz bei akuter und chronischer Sinusitis durch eine Langzeittherapie mit topischen Steroiden ergänzt werden. Dabei seien eine starke lokale Wirkung einerseits und eine geringe systemische Bioverfügbarkeit andererseits, wie bei Mometason, von großer Wichtigkeit.

Laut Gerd Rasp (HNO-Klinik der LMU München) sind für die Behandlung der entzündlichen Komponente der chronischen Sinusitis niedrig dosierte topische Steroide der einzige sinnvolle Ansatz, zumal die bei akuter Sinusitis indizierten abschwellenden Nasentropfen nebenwirkungshalber langfristig nicht in Frage kommen.

\section{... als auch bei Nasenpolypen}

Während einige Autoren Allergien gegen Aeroallergene für die Entstehung der Polyposis nasi verantwortlich machen, werden von anderen die ASS-Intoleranz sowie neuerdings auch Pilze und bakterielle Infekte in den Vordergrund gestellt. Der ödematöse Zustand der Nasenpolypen kommt durch eine entzündliche Plasmaexudation bzw. einen erhöhten Albumingehalt zustande, erklärte Claudia Rudack (HNO-Klinik der Universität Münster). So wie bei der der Behandlung Sinusitis mit Mometason eine deutliche Reduktion der Eosinophilen und des Albumins im Nasensekret zu beobachten ist, so ergibt sich auch bei Polypen eine Therapieoption. Sowohl systemische als auch topische Steroide seien in der Therapie der Polyposis nasi und bei der postoperativen Rezidivprophylaxe etabliert.

Symposium „Nasonex ${ }^{\circledR}$ wirkungsvoll bei Sinusitis und Polypen“ Malelane, 2001. Veranstalter: Essex Pharma.

\title{
Keramikfräse ersetzt Patientenenergie
}

Fing CKW-haltige Dosieraerosole waren lange Zeit Standard in der ambulanten Behandlung von Asthma. Mit der Entdeckung der zerstörerischen Wirkung der FCKW-Treibgase auf das Ozon der Stratosphäre etablierten sich zunehmend Pulverinhalatoren als alternative Inhalationsgeräte.

Pulverinhalatoren kommen ohne Treibgase aus, die Freisetzung erfolgt in der Regel durch den Atemzug der Patienten. Dies kann allerdings in Einzelfällen zu Problemen führen, denn der mikronisierte Wirkstoff muss immer aus einer „Ruhelage" heraus mobilisiert werden ein Vorgang, für den eine definierte Energie nötig ist.

Diese zur Mobilisation und Desagglomeration benötigte Energie muss der Patient selbst über seinen inspiratorischen Atemfluss zur Verfügung stellen. Ist der Atemfluss zu schwach, kann nicht genügend Kraft aufgebracht werden, um das Pulver zu aktivieren. Der Patient profitiert damit nur suboptimal von der Therapie.

Einen neuen Weg der Wirkstofffreisetzung in Pulverinhalatoren wurde mit der Entwicklung des MAGhaler ${ }^{\circledR}$ beschritten. Bei diesem System liegt der Wirkstoff in Form einer hochverdichteten, wenig feuchtigkeitsempfindlichen Tablette vor. Das Gerät ist mit einer gehärteten Keramikfräse ausgestattet, die auf Knopfdruck durch den Patienten, angetrieben durch einen mechanischen Federmotor, die Einzeldosis in reproduzierbarer Menge abreibt.

$\mathrm{Da}$ die Freisetzung nicht aus einer "Ruhelage des Pulvers" wie bei herkömmlichen Geräten geschieht und damit keine energieverbrauchende Desagglomeration mehr nötig ist, atmen auch Patienten mit einem schwachen Atemzugsvolumen die volle therapeutische Dosis ein.
Die Äquivalenz des MAGhaler ${ }^{\circledR}$ Systems mit FCKW-haltigen Dosieraerosolen konnte in mehren Studien sowohl für Budesonid wie auch Salbutamol gezeigt werden. Hinsichtlich der Akzeptanz und Präferenz bei den Patienten ergaben sich jedoch Vorteile für den neuen Pulverinhalator. Auch Kinder dürften von dem MAGhaler ${ }^{\circledR}$ profitieren, vor allem, weil mit weniger Koordinationsschwierigkeiten zu rechnen ist.

Die Antriebseinheit MAGhaler ${ }^{\circledR}$ kann über zwei Jahre verwendet werden, ausgetauscht wird lediglich die Wirkstoffeinheit mit Mundstück, die MAGtab $^{\circledR}$ mit 300 Einzeldosierungen. Erhältlich sind voraussichtlich ab April 2001 die Wirkstoffe Salbutamol (Salmundin $^{\circledR}$ MAGtab $^{\circledR}$ ) und Budesonid (Respicort ${ }^{\circledR}$ MAGtab $\left.^{\circledR}\right)$.

Fleischer W, Mitfessel H, Kunkel G, Lecheler J. Einführungskonferenz „Moderne Asthma-Therapie: Einen Atemzug voraus mit dem MAGhaler ${ }^{\circledR}$ ", Veranstalter: Mundipharma GmbH, München, 2001. 